\title{
Observation of Gamma-Ray Bursts and short duration transients with Fermi Large Area Telescope
}

\author{
Giacomo Vianello* \\ Stanford University \\ E-mail: giacomovestanford.edu
}

\section{Nicola Omodei}

Stanford University

\section{Frederic Piron}

Laboratoire Univers et Particules de Montpellier (CNRS/IN2P3)

\section{Soebur Razzaque}

University of Johannesburg

\section{on behalf of the Fermi/LAT collaboration}

\begin{abstract}
Observations of Gamma-Ray Bursts with the Fermi Large Area Telescope have prompted theoretical advances and posed big challenges. The first Fermi/LAT GRB catalog, based on 35 detections collected in the first 3 years of observations, established many interesting features and opened new questions. The record-breaking GRB 130427A took the challenge even further, underlining even more the need for new theoretical developments. After a brief review of the main results, we present a new detection algorithm that is $40 \%$ more efficient than the previous one: with this procedure, as well as a new and improved event analysis ("Pass 8"), the Fermi/LAT now detects more than $100 \mathrm{GRBs}$ in the first 6.5 years of observations. The characterization of this sample is ongoing. In addition, we are developing a blind search algorithm aimed to detect short-duration transients ( $<3$ hours). We briefly describe such development and its high potential for discoveries.
\end{abstract}

3rd Annual Conference on High Energy Astrophysics in Southern Africa-HEASA2015,

18-20 June 2015

University of Johannesburg, Auckland Park, South Africa

* Speaker. 


\section{Introduction}

Fermi/LAT observations are uncovering new and unexpected properties of the high-energy emission from Gamma-Ray Bursts (GRBs) shedding light on their physical mechanisms, such as particle acceleration and emission processes in ultra-relativistic jets. The first LAT GRB catalog [1] contained 35 bursts, among which 28 have been detected above $100 \mathrm{MeV}$ with the standard likelihood analysis and 7 with the LAT Low-Energy technique (LLE). It established new high-energy features of GRBs, namely:

- Additional power-law component during the prompt emission: the prompt emission spectrum of most GRBs has been successfully described in the past with the Band function [2]. In many bright GRBs observed by the LAT an additional power-law component is required to account for high-energy data.

- Delayed onset: the emission above $100 \mathrm{MeV}$ is systematically delayed with respect to the low-energy emission seen in the keV-MeV energy range.

- Extended duration: the emission above $100 \mathrm{MeV}$ is also systematically longer than the $\mathrm{keV}-$ $\mathrm{MeV}$ prompt emission. The luminosity above $100 \mathrm{MeV}$ decreases smoothly with time as $t^{-1}$, pointing to a different physical origin with respect to the highly variable and spiky prompt emission.

Early afterglow models $[3,4]$ could explain the observed temporal decay, the delayed onset as the outflow deceleration time scale, and the apparent lack of variability in most LAT-detected GRBs. Hadronic models could explain these features as well: the onset delay could be the time to produce electromagnetic cascades $[5,6,7]$ or the time required to accelerate, accumulate, and cool down relativistic protons via synchrotron emission in a very strong magnetic field [8]. Furthermore, energy-dependent delays would be expected in proton-synchrotron models where the cooling break shifts to lower energies at later times. On the other hand, we have analyzed the record-breaking GRB 130427A finding that, while several features of the "prompt" emission are in agreement with some internal-shocks models, other key details such as the estimated Lorentz factors for the colliding shells contradict expectations, possibly calling for different scenarios [9]. Also, the maximum energy of the photons in conjunction with a featureless high-energy light curve rules out both synchrotron and Synchrotron Self Compton from Fermi-accelerated particles as emission mechanism, a radical departure from the standard external-shock model for the LAT emission [10]. New observations and insights are hence needed to foster further theoretical developments and overcome these difficulties.

Our new specialized analysis described in Sect. 2 allows the detection of faint high-energy GRB counterparts, increasing the efficiency of detection by more than $50 \%$ and, when used in conjuction with the new "Pass 8" event selection, yielding more than 100 bursts over the time span of the Fermi mission. The analysis and characterization of this new sample is in progress. When completed, it will provide the needed new insights. In particular, we will be able to settle some open questions which we could not firmly establish in the first catalog due to the limited statistics:

- The existence of a separate population of hyper-energetic events, characterized by a ratio between high- and low-energy fluence much larger than the others. 
- The high-energy emission for all but 2 GRBs in the sample decays as $t^{-1}$ at late times, which in the context of the fireball model favors an adiabatic expansion. The other two GRBs instead decay as expected from a radiative regime $\left(t^{-1.5}\right)$ [11, 12], but they suffered from less-than-optimal observing conditions. Is there a class of truly radiative fireballs?

- One of the GRBs in the sample presented a high-energy cutoff in the extra power-law component, while another one in the low-energy Band component. How common are spectral cutoffs? Do bursts with a cutoff have any other peculiar feature?

Analyzing the new enlarged sample will answer these questions, and will uncover new features.

\section{New triggered search for Gamma-Ray Bursts}

Before the launch of Fermi it was estimated that the LAT would have observed 10-12 GRBs/year above $100 \mathrm{MeV}$ and 6-8 above $1 \mathrm{GeV}$ [13]. During the first 3 years, however, observations were slightly below such expectations [1,14]. Recently we developed a new search algorithm for GRBs, which is now up and running 24/7. It provides $45 \%$ more detections than the algorithm used for the catalog when using Pass 7 data, and 60\% more when using the newly-developed Pass 8 data. This improvement was achieved exploiting the results of the first LAT GRB catalog, presented in Sect. 1. The new algorithm consists of 10 searches running in parallel over time intervals logarithmically spaced from the trigger time up to $10 \mathrm{ks}$ post-trigger. For each of these time intervals, the steps of the new algorithm are the following:

1. A trigger is received either in real-time through the GCN system ${ }^{1}$ or during an off-line analysis. In real-time most of the triggers come from the Fermi Gamma-ray Burst Monitor (GBM), although also triggers from Swift, INTEGRAL and other space observatories are received and processed.

2. If the trigger comes from the GBM, a "finding map" is produced to account for the position uncertainty. It has been recently reported that the GBM localizes GRBs with a systematic error of up to $15^{\circ}$ [15]. Since this systematic error dominates over the statistical one, we always use finding maps of $30 \times 30 \mathrm{deg}$. The finding map is essentially a TS map similar to the one produced by the tool gttsmap. Indeed, a grid in equatorial coordinates and with a spacing of $0.7 \mathrm{deg}$ is created covering the finding map. For each point on the grid, a likelihood analysis is performed and computes the TS of a new source at that position. Points in the grid having less than 3 photons within $10 \mathrm{deg}$ are not considered. This avoids running a likelihood analysis, which is computing-intensive, in regions of the finding map where there is no possibility of detecting a new point source. When all points have been processed, the maximum of the TS in the map is considered as the best guess for the position of the new transient, and marked for further analysis. Note that if the trigger comes from Swift or INTEGRAL, this step is not executed, since their localization errors are much smaller than the typical size of the LAT PSF.

\footnotetext{
${ }^{1}$ Gamma-Ray Coordinate Network, http://gcn.gsfc.nasa.gov/
} 
3. The position of the candidate transient is optimized with the tool gtfindsrc.

4. A new likelihood analysis is performed on the best position found. If the TS from this final analysis is above a certain threshold, we consider it a new detection.

5. If running in real time, the results from the analysis are used by Burst Advocates to disseminate alerts to the community through GCNs.

In all likelihood analyses involved in the sequence described above, the likelihood model consists of the Galactic template and the Isotropic template provided by the Fermi collaboration, plus all point sources from the 3FGL source catalog [16]. While the normalization of the two templates are left free to vary, all parameters for the 3FGL sources are kept fixed. If the algorithm finds a new candidate source with a position compatible with one of the 3FGL sources, further analysis are performed manually to distinguish between real transients and other phenomena such as AGN flares. The algorithm does indeed trigger on flaring sources, which are also found by other real-time algorithms such as ASP [13] and FAVA [17].

The procedure involves a certain number of trials, which might appear large. However, all the time windows are overlapping and involve the same region on the sky, therefore these trials are not independent at all, with many photons present in many of the time windows. Thus, the effective number of trials is rather small. While the evaluation of the trial factor is still in progress, preliminary simulations indicate that a $5 \sigma$ detection corresponds to $\mathrm{TS} \sim 28$.

A first run of the new algorithm on $\sim 5$ years of Pass 8 data returned 86 detections. Adding the $\sim 20$ LLE-only detections, we have already reached the milestone of 100 LAT-detected GRBs. Given the current rate of detections we also expect to exceed 100 likelihood detections within the year, which significantly exceeds pre-launch expectations. As expected, the new detections populate the lower part of the fluence distribution (Fig. 1), demonstrating that the new algorithm and "Pass 8 " improve the sensitivity of the search.

\section{New blind search for transients}

The current picture of GRBs admits the possibility of a high-energy signal in absence of a low-energy counterpart. Orphan high-energy detections could be expected from very highredshift GRBs, such as those from population III stars [18] which are too faint to trigger the GBM, or from GRBs whose jets point away from the Earth, but becomes visible during the afterglow phase as the fireball decelerates. We therefore have developed and implemented a new blindsearch analysis. While blind-search algorithms have been running since the launch of Fermi [13], they are not optimized for short and relatively faint transients, and indeed they do not trigger on GRBs except for exceptionally bright ones. Our new analysis focuses on time scales shorter than 6 hours, and it covers a region of the parameter space left unexplored up to now. This new analysis allows the detection of short and faint transients, to which current algorithms are not sensitive to (Fig. 2). In addition to GRBs, primordial black holes (PBHs) with a mass around $5 \times 10^{14}$ $\mathrm{g}$ and temperatures around $50-500 \mathrm{GeV}$ are expected to produce a burst of photons in the LAT 


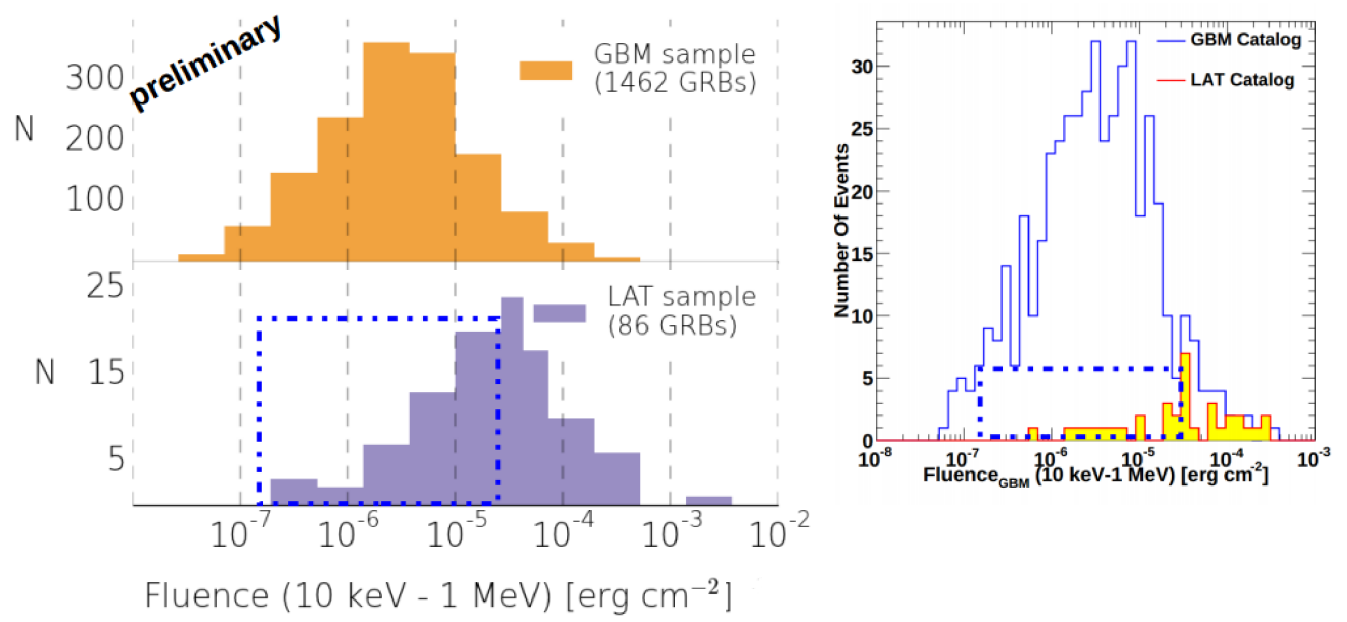

Figure 1: Left panel: low-energy fluence distribution for the whole GBM sample (upper panel) and for the sample detected with the new algorithm in LAT data (lower panel). Right panel: similar plot from the first LAT GRB catalog [1]. The blue dashed line marks the region where most of the new detections lie, showing the increased sensitivity of the new algorithm with respect to the old one.

energy range with a duration smaller than one hour ${ }^{2}$. Unlocking a new region in the parameter space opens also the possibility for unexpected new discoveries, as it recently happened with the extra-galactic Fast Radio Transients [19].

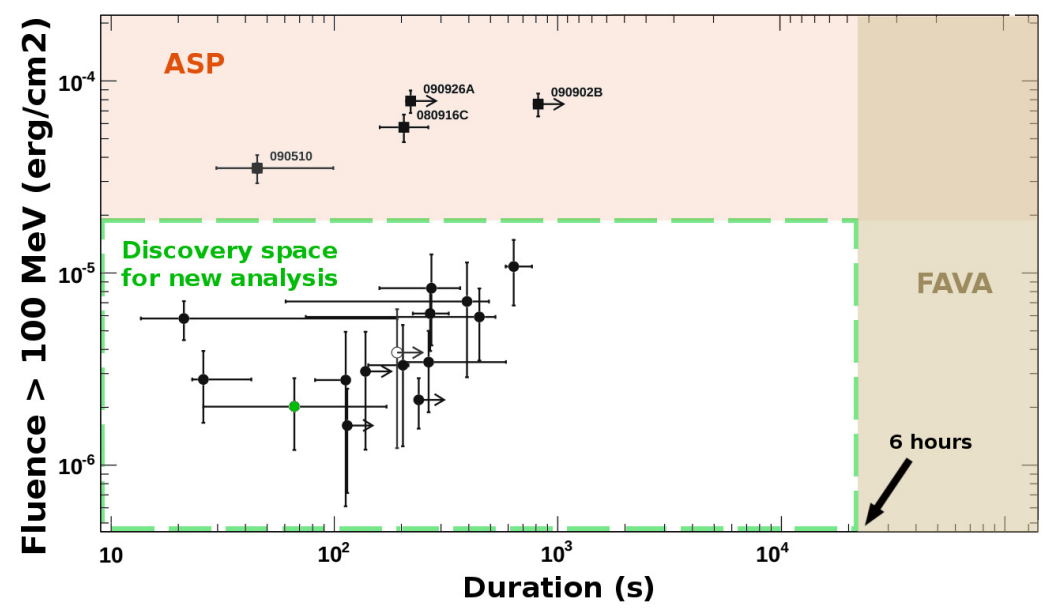

Figure 2: Duration vs. fluence from the LAT GRB catalog [1]. Among all these GRBs found by the triggered search, existing blind searches (ASP [13] and FAVA [17]) have triggered only on the 4 brightest. Our blind search instead triggered even on the faint GRB 090217 (in green), and it is indeed exploring an uncharted discovery space.

The new algorithm considers the data in chunks of 24 hours, and divides the sky in 1712

\footnotetext{
${ }^{2}$ See for example http://fermi.gsfc.nasa.gov/science/mtgs/symposia/2014/program/16_MacGibbon.pdf and references therein
} 
overlapping circular regions with a radius of $5.2^{\circ 3}$, shifted by half radius. For each region we run a modified version of the Bayesian Blocks algorithm [20]. The standard BB algorithm (sBB) finds time intervals with perceptively constant count rates, without any assumption about their duration. Since the LAT operates nominally in survey mode, the count rate in each region is continuously changing due to the change in exposure, and the sBB algorithm finds many time intervals following such variations. Instead, our modified version $(\mathrm{mBB})$ takes into account the variable exposure, and
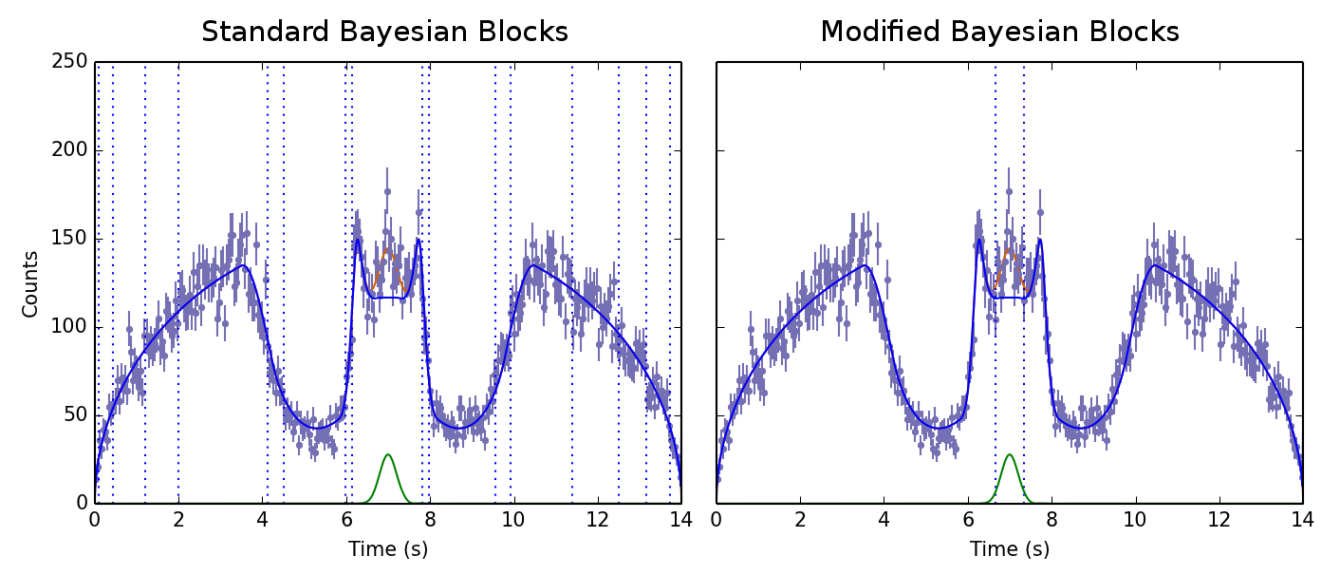

Figure 3: Simulation of a transient (green line) over a time-varying background (blue line). The standard Bayesian Block algorithm finds many intervals (left panel, dashed lines) following the variations in the background, and misses the transient. The modified BB algorithm (right panel) finds only the transient.

finds statistically significant excesses over the background. This is illustrated through a simulation in Fig.3. Practically we estimate the background rate by convolving the time-varying exposure with the information in the LAT source catalog and in the templates for the diffuse emission [16]. After running $\mathrm{mBB}$ on all the regions, we run a normal likelihood analysis on the candidates having a high statistical significance. Running the algorithm on the first 6 months of Pass 7 data $^{4}$ we detected several excesses with duration from less than a second to several thousand seconds. In particular we found almost all GRBs already seen with the triggered analysis, both long and short, plus several known flares from AGNs. This confirms that the algorithm is working very well and is indeed time-scale-agnostic. In particular, the detection of the relatively faint GRB 090217 confirms that we are exploring a region in the parameter space inaccessible by the existing algorithms (Fig. 2). In conclusion, with this new algorithm coming online we are about to open a new discovery region for short-duration transients.

\section{Acknowledgments}

The Fermi LAT Collaboration acknowledges generous ongoing support from a number of agencies and institutes that have supported both the development and the operation of the LAT as well as scientific data analysis. These include the National Aeronautics and Space Administration

\footnotetext{
${ }^{3}$ corresponding to the $68 \%$ containment of the LAT PSF for P7REP_SOURCE_V15 class

${ }^{4}$ P7REP_SOURCE_V15 class
} 
and the Department of Energy in the United States, the Commissariat à l'Energie Atomique and the Centre National de la Recherche Scientifique / Institut National de Physique Nucléaire et de Physique des Particules in France, the Agenzia Spaziale Italiana and the Istituto Nazionale di Fisica Nucleare in Italy, the Ministry of Education, Culture, Sports, Science and Technology (MEXT), High Energy Accelerator Research Organization (KEK) and Japan Aerospace Exploration Agency (JAXA) in Japan, and the K. A. Wallenberg Foundation, the Swedish Research Council and the Swedish National Space Board in Sweden.

Additional support for science analysis during the operations phase is gratefully acknowledged from the Istituto Nazionale di Astrofisica in Italy and the Centre National d'Études Spatiales in France.

\section{References}

[1] Ackermann, M. et al, The First Fermi-LAT Gamma-Ray Burst Catalog, ApJS 209 (Nov., 2013) 11, [1309.4899].

[2] Band, D. et al, BATSE observations of gamma-ray burst spectra. I - Spectral diversity, ApJ 413 (Aug., 1993) 281-292.

[3] Kumar, P. and Barniol Duran, R., On the generation of high energy photons detected by the Fermi Satellite from gamma-ray bursts, ArXiv e-prints (May, 2009).

[4] Ghisellini, G. et al, GeV emission from gamma-ray bursts: a radiative fireball?, MNRAS 403 (Apr., 2010) 926-937, [0910.2459].

[5] Dermer, C.D. and Atoyan, A., Ultra-high energy cosmic rays, cascade gamma rays, and high-energy neutrinos from gamma-ray bursts, New Journal of Physics 8 (July, 2006) 122, [arXiv:astro-ph/0606629].

[6] Gupta, N. and Zhang, B., Prompt emission of high-energy photons from gamma ray bursts, MNRAS 380 (Sept., 2007) 78-92, [arXiv:0704.1329].

[7] Asano, K. and Inoue, S., Prompt GeV-TeV Emission of Gamma-Ray Bursts Due to High-Energy Protons, Muons, and Electron-Positron Pairs, ApJ 671 (Dec., 2007) 645-655, [0 705 . 2910 ].

[8] Razzaque, S., Dermer, C.D. and Finke, J.D., Synchrotron radiation from ultra-high energy protons and the Fermi observations of GRB 080916C, ArXiv e-prints 0908.0513 (Aug., 2009) .

[9] Preece, R. et al, The first pulse of the extremely bright grb 130427a: A test lab for synchrotron shocks, Science 343 (2014) 51-54, [http://www.sciencemag.org/content/343/6166/51.full.pdf].

[10] Ackermann, M. et al, Fermi-lat observations of the gamma-ray burst grb 130427a, Science 343 (2014) 42-47, [http: / /www.sciencemag.org/content/343/6166/42.full.pdf].

[11] Blandford, R.D. and McKee, C.F., Fluid dynamics of relativistic blast waves, Physics of Fluids 19 (Aug., 1976) 1130-1138.

[12] Katz, J.I. and Piran, T., Persistent Counterparts to Gamma-Ray Bursts, ApJ 490 (Dec., 1997) 772.

[13] Band, D.L. et al, Prospects for GRB Science with the Fermi Large Area Telescope, ApJ 701 (Aug., 2009) 1673-1694, [0906.0991].

[14] Guetta, D., Pian, E. and Waxman, E., FERMI constraints on the high energy, 1 GeV, emission of long gamma ray bursts, A\&A 525 (Jan., 2011) A53, [1003.0566]. 
[15] Connaughton, V. et al, Localization of Gamma-Ray Bursts using the Fermi Gamma-Ray Burst Monitor, ArXiv e-prints (Nov., 2014) , [1411.2685].

[16] The Fermi-LAT Collaboration, Fermi Large Area Telescope Third Source Catalog, ArXiv e-prints (Jan., 2015), [1501.02003].

[17] Ackermann, M. et al, The Fermi All-sky Variability Analysis: A List of Flaring Gamma-Ray Sources and the Search for Transients in Our Galaxy, ApJ 771 (July, 2013) 57, [1304.6082].

[18] Mészáros, P. and Rees, M.J., Delayed GEV Emission from Cosmological Gamma-Ray Bursts - Impact of a Relativistic Wind on External Matter, MNRAS 269 (July, 1994) L41+, [arXiv:astro-ph/9404056].

[19] Thornton, D. et al, A Population of Fast Radio Bursts at Cosmological Distances, Science 341 (July, 2013) 53-56, [1307.1628].

[20] Scargle, J.D. et al, Studies in Astronomical Time Series Analysis. VI. Bayesian Block Representations, ApJ 764 (Feb., 2013) 167, [1207.5578]. 